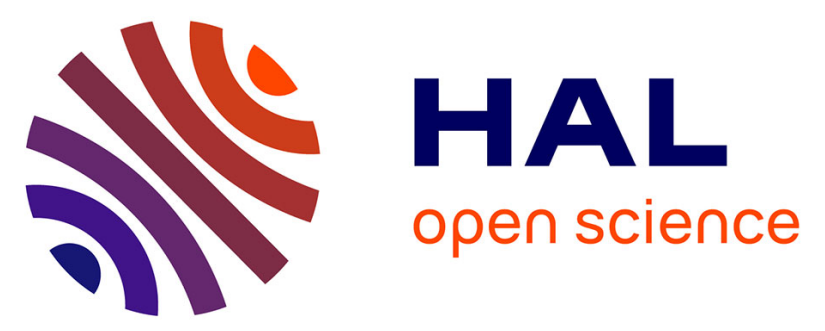

\title{
Ultrasensitive Interferons quantification in idiopathic inflammatory myopathies serve as biomarkers of activity in dermatomyositis and anti-synthetase syndrome
}

Bolko Loïs, Celine Anquetil, Alba Llibre, Solene Maillard, Damien Amelin, Karim Dorgham, Vincent Bondet, Oceane Landon-Cardinal, Segolene Toquet, Kuberaka Mariampillai, et al.

\section{To cite this version:}

Bolko Loïs, Celine Anquetil, Alba Llibre, Solene Maillard, Damien Amelin, et al.. Ultrasensitive Interferons quantification in idiopathic inflammatory myopathies serve as biomarkers of activity in dermatomyositis and anti-synthetase syndrome. 2021. pasteur-03252308

\section{HAL Id: pasteur-03252308}

https: / hal-pasteur.archives-ouvertes.fr/pasteur-03252308

Preprint submitted on 7 Jun 2021

HAL is a multi-disciplinary open access archive for the deposit and dissemination of scientific research documents, whether they are published or not. The documents may come from teaching and research institutions in France or abroad, or from public or private research centers.
L'archive ouverte pluridisciplinaire HAL, est destinée au dépôt et à la diffusion de documents scientifiques de niveau recherche, publiés ou non, émanant des établissements d'enseignement et de recherche français ou étrangers, des laboratoires publics ou privés.

\section{(c)(1)}

Distributed under a Creative Commons Attribution| 4.0 International License 


\section{Ultrasensitive Interferons quantification in idiopathic inflammatory myopathies serve as biomarkers of activity in dermatomyositis and anti-synthetase syndrome}

\section{CURRENT STATUS: POSTED}

Research Square

BOLKO Loïs

Hôpital Maison Blanche

- Ibolko@chu-reims.frCorresponding Author

ORCiD: https://orcid.org/0000-0002-0186-5570

Celine Anquetil

Assistance publique-Hopitaux de Paris, Department of internal medicine and clinical immunology, National Reference center for Rare NeuroMuscular disorders, Pitié-Salpêtrière Hospital, Paris, France

Alba llibre

Immunobiology of dendritic cells, Inserm 1223, Institut pasteur, Paris, France

Solene Maillard

Sorbonne Universite, INSERM, Association Institut de Myologie, Center of research of Myology, UMRS974, Paris, France

Damien Amelin

Sorbonne Universite, Inserm, Association instut de myologie, Center of research in Myology, UMRS 974, Paris France

Karim Dorgham

Sorbonne Universite, Inserm, centre d'immunologie et maladies infectieuses (CIMI-Paris), assistance publique- hopitaux de paris, Hopital pitié Salpêtrière, 75013 Paris

Vincent Bondet

Immunobiology of dendritic cells, inserm U1223, Institut pasteur, paris, france

Oceane Landon-Cardinal

Department of medicine, University of Montreal, Canada, Division of Rheumatology, Centre hospitalier de I'université de Montreal (CHUM), Canada, CHUM Research Center

Segolene Toquet

Assistance Publique - Hopitaux de Paris, Department of internal medicine and clinical immunology, 
Kuberaka Mariampillai

Assistance Publique - Hopitaux de Paris, department of internal medicine and clinical immunology, National reference center for rare neuromuscular disorders, Pitié-Salpêtrière Hospital, Paris, France

Alexandrine Mahoudeau

Sorbonne Universite, Inserm, Association institut de Myologie, Center of research in Myology, UMRS 974, Paris, France

Baptiste Hervier

Assistance Publique - Hopitaux de Paris, department of internal medicine and clinical immunology, National reference center for rare neuromuscular disorders, Pitié-Salpêtrière Hospital, paris, france

Mathieu Rodero

Chimie et biologie, Modélisation et immunologie pour la thérapie (CBMIT), Université Paris descartes, CNRS, UMR 8601, Paris, France

\section{Guy Gorochov}

Sorbonne Universite, Inserm, Centre d'immunologie et des maladies infectieuses (CIMI-Paris), Assistance publique - Hopitaux de Paris, Hopital de la pitié Salpêtrière, 75013 Paris france

Darragh Duffy

Immunobiology of dendritic cells, INSERM U1223, Institut pasteur, Paris, France

Olivier Benveniste

Assistance Publique - Hopitaux de Paris, department of clinical immunology and internal medicine, National reference center for neuromuscular disorders, Pitié-Salpêtrière hospital, Paris, France

Yves Allenbach

Assistance publique - Hopitaux de Paris, department of clinical immunology and internal medicine, National reference center for rare neuromuscular disorder, Paris, France

\section{DOI:}

$10.21203 / \mathrm{rs} .2 .23943 / \mathrm{v} 1$

\section{SUBJECT AREAS}

Rheumatology

\section{KEYWORDS}

Idiopathic inflammatory myopathies, Interferon, Biomarkers, Disease activity 
Abstract

Objectives Inflammatory idiopathic myopathies (IIM) are a heterogeneous group of disorders, ranging from a muscle-specific autoimmune disease to a systemic one that are difficult to assess. Recent insights into IIM pathogenesis highlighted the role of interferon (IFN) in the pathophysiology. The aim of this study was to test if IFN serum levels can a use as a biomarker of disease activity in IIM.

Methods IFN type I and II were measured using an ultrasensitive detection technology and assess the potential of IFN.

Results One hundred and fifty-two patients (dermatomyositis (DM); $n=50$, anti-synthetase syndrome (ASyS); $n=46$, immune-mediated necrotizing myopathy (IMNM); $n=32$, inclusion body myositis (IBM); $n=24)$ and 33 age-matched healthy donors were included. IFN- $\alpha$ levels were higher only in DM (0.07 $\mathrm{pg} / \mathrm{ml}[0.03-0.23], \mathrm{p}<0.005)$ and ASyS groups $(0.07[0.02-0.16], \mathrm{p}<0.05)$ compared with controls (0.02 [0.01-0.05]). IFN- $\beta$ was increased only in DM and IFN- $\gamma$ among all IIM. IFN- $\alpha$ levels were correlated with disease activity in DM $(r=0.76, p<0.0001)$. The predictive accuracy of IFN- $\alpha$ level to discriminate active and non-active disease was excellent as reflected by an area under the ROC-curve of 0.88 . Using an IFN- $\alpha$ level cut-off above $0.11 \mathrm{pg} / \mathrm{ml}$, the sensitivity was $75 \%$ and the specificity was $96 \%$ in DM patient. IFN- $\alpha$ and IFN- $\gamma$ were correlated with disease activity in ASyS groups ( $r=0.55$ and $r=0.46 p<0.05))$.

Conclusions IFNs are promising biomarker for DM and ASyS disease activity.

\section{Key Messages}

What is already known about this subject?

Idiopathic inflammatory myopathies are a heterogeneous group of diseases combining muscle and extra-muscular manifestations difficult to assess

Interferon signature correlates with dermatomyositis (DM) disease activity

What does this study add?

Seric levels of IFN- $\alpha$ and $\beta$ (type I IFN) are increased in DM and IFN- $\alpha$ and -g in anti-synthetase syndrome (ASyS)

IFN- $\alpha$ is well correlated with disease activity and permit to discriminate active from inactive patients How might this impact on clinical practice or future developments?

IFN- $\alpha$ can be considered as a biomarker of disease activity in DM and ASyS IFNs increased levels in DM and in ASyS open new avenue for targeted treatment Introduction 
Idiopathic inflammatory myopathies (IIM) are a heterogenous group of autoimmune diseases including four main groups: dermatomyositis (DM), anti-synthetase syndrome (ASyS), immune-mediated necrotizing myopathies (IMNM), and inclusion body myositis (IBM) (1,2). IIM can either be a musclespecific autoimmune disease (IBM and IMNM) or systemic involving mainly the skin, the joints and/or the lungs (DM and ASyS). The development of tools assessing disease activity is crucial in daily clinical practice to improve patients' care, as well as improving the design of randomized clinical trials. International Myositis Assessment and Clinical Studies Group developed a disease activity core set measures permitting to define an improvement using a total improvement score calculated with two timepoints measures (3). Nevertheless, reliable biomarkers are needed to assess the disease activity at one given timepoint. While creatine kinase (CK) level (one core set measure) is well correlated with IMNM disease activity (4), it may lack sensitivity in ASyS or DM patients (5). Interferons (IFN) are involved in the pathophysiology of IIM in which an overexpression of IFNstimulated genes is observed $(6,7)$. There are three main types of IFN. The type I IFN (IFN- $\alpha$ and $\beta$ ) blood levels are increased in DM $(8,9)$ whereas the role of type I IFN in ASyS has not been yet clarified. In IBM, large amounts of type II IFN (IFN-Y) are produced by activated CD8 + T cells that play key role in muscle fibers damages $(10,11)$. Serum level of type II IFN have not been evaluated in IIM yet. In addition, a new ultrasensitive technology has recently been developed and permits to detect very low level of proteins suitable for cytokine detection at femtomolar concentration such as IFN- $\alpha$ (12).

The aim of this study was to assess the potential of type I and II IFN, using an ultrasensitive digital ELISA technology, as a new blood biomarker of activity for IIM especially in DM and ASyS.

\section{Methods}

\section{Patients And Sera}

Patients were prospectively enrolled between 2011 and 2018 in a tertiary center of IIM (PitiéSalpêtrière Hospital, Paris, France). They fulfilled the ACR/EULAR classification criteria for myositis (13). Patients were classified into four categories: IBM (Lloyd's criteria (14)), IMNM (ENMC 2017 (15)), ASyS in presence of anti-synthetase antibody and DM (1-14). 
Sera were collected at diagnosis and/or during the follow-up and were rapidly $(<3 \mathrm{~h}$ ) frozen after one centrifugation. All the sera were thawed only once to avoid potential freeze/thaw effects. Patients who had increased dose of corticosteroids ( $>0.5 \mathrm{mg} / \mathrm{kg}$ and/or pulses) the week before the sampling were excluded as it may rapidly abrogate the IFN levels (16). Moreover, patients with active infectious diseases (e.g. flew or viral B hepatitis) were excluded. Thirty-three age-matched healthy donors (HD) from a French blood bank were used as negative controls.

The screening for Myositis-Specific Antibody (MSA) was performed with different line blot commercial assays as previously reported (1) including anti-melanoma differentiation-associated protein 5 (antiMDA5), -transcription intermediary factor- $\gamma$ (anti-Tif1 $\gamma$ ), -complex nucleosome remodeling histone deacetylase (anti-Mi2), -nuclear matrix protein 2 (anti-NXP2) and -SUMO-activating enzyme subunit 1 (anti-SAE1) for DM; -histidyl-ARNt synthetase (anti-Jo1), -threonine-ARNt synthetase (anti-PL7), alanine-ARNt synthetase and -glycine-ARNt synthetase (anti-EJ) for ASyS; -signal recognition particle (anti-SRP) and - 3-hydroxy-3-methylglutaryl-coenzyme A reductase (anti-HMGCR) for IMNM. Myositisassociated autoantibodies (MAA) were screened by commercial assay including anti-Ro52, anti-Ro60, anti-La, anti-cyclic citrullinated peptide, and rheumatoid factor.

\section{Disease Activity Assessment}

Using International Myositis Assessment and Clinical Studies Group core set measures the following assessments were performed: Manual Muscle Testing 8 (MMT8), Creatine Kinase (CK) level, Myositis Disease Activity Assessment Tool (MDAAT), Physician Global Activity (PGA). Disease activity was assessed at the time of blood collection and the result was represented in a numeric scale (from 0 to 10; 0 corresponding to the remission without treatment and 10 the maximum disease activity).

\section{IFN Serum Measurement By Single Molecule Array (Simoa ${ }^{\circledR}$ )}

IFN- $\alpha$ and IFN- $\gamma$ serum concentrations were measured using the high sensitivity Simoa ${ }^{\circledR}$ technology (Digital ELISA technology) (Quanterix SimoaTM IFN $\alpha$ Reagent Kit, Lexington, MA, USA and Quanterix SimoaTM IFNY Reagent Kit, Lexington, MA, USA) according to the manufacturer protocols and as previously reported (9,17-19).

The limit of detection (LOD) was $0.0035 \mathrm{pg} / \mathrm{ml}$ for IFN- $\alpha$ and $0.026 \mathrm{pg} / \mathrm{ml}$ for IFN- $\gamma$. The positivity 
threshold was defined as the mean plus three times the standard deviation of the 33 healthy donors (HD) and was $0.22 \mathrm{pg} / \mathrm{ml}$ for IFN- $\alpha$ and $1.97 \mathrm{pg} / \mathrm{ml}$ for IFN- $\gamma$.

For IFN- $\beta$ serum quantification, we developed a homebrew assay using Simoa ${ }^{\circledR}$ reagents and mAbs from (PBL Assay Science, Piscataway, NJ, USA), as recently described (Llibre et al, In Press). The LOD was calculated by the mean value of the blank plus two times the standard deviation (positivity at $95 \%$ confidence) calculated on logarythmic values and was $1.24 \mathrm{pg} / \mathrm{ml}$ and the positivity threshold was defined by the mean plus three times the standard deviation of the HD and was $2.50 \mathrm{pg} / \mathrm{ml}$. Statistical analysis

Quantitative variables were expressed as median with inter-quartile range, and numbers with proportions for categorical variables. Multiple comparisons were performed using Kruskal-Wallis test then Dunn's post-hoc test for quantitative data. To analyse the correlation between IFN and disease activity assessed by the PGA, we performed Spearman's rank correlation tests using Graphpad prism 5. Positive threshold to discriminate active from inactive patient was assessed by ROC curve analysis. We used the point of the curve nearest the top of the left-hand corner to minimise both the number of false-positive and false-negative results. CK and IFN values were transformed through a base-10 logarithm for analysis. After verifying the absence of multicolinearity, we included IFN- $\alpha$, IFN- $\gamma$ and CK levels in binary multivariate logistic regression to determine the association with disease activity (binary outcome using PGA $>5$ to define active patients). $\mathrm{P}<0.05$ was considered statistically significant. Multivariate statistical analyses were realized with R program version R-3.6.0.

\section{Ethical}

Written informed consent from each study patient and approval by local Ethics Committee (CPP Ile De France VI (2013-12-19), CCTIRS (N¹4.323) and CNIL (AR158656)) were obtained.

\section{Results}

\section{Patients' characteristics}

One hundred fifty-two IIM patients (DM, $n=50 ;$ ASyS, $n=46 ;$ IMNM, $n=32$ and IBM, $n=24$ ) and 33 healthy donors were included. Main patients' characteristics are shown in Table 1. Table I: Patients' characteristics at blood sampling timepoint 


\begin{tabular}{|c|c|c|c|c|c|c|}
\hline Diagnosis & DM & ASyS & IMNM & IBM & Total & $p$ \\
\hline n (\%) & $50(33)$ & $46(30)$ & $32(21)$ & $24(16)$ & $152(100)$ & - \\
\hline Age (year) & $53.2 \pm 15.4$ & $48.6 \pm 14.5$ & $49.6 \pm 19.1$ & $69.3 \pm 8.3$ & $53.6 \pm 16.6$ & $p<0.001$ \\
\hline MSA, n (\%) & $35(70)$ & $46(100)$ & $32(100)$ & $12(100)$ & 125 & - \\
\hline MAA, n (\%) & $13(26)$ & $37(80)$ & $9(28)$ & $3(13)$ & 62 & - \\
\hline MMT8 (0-150) & 142 [126- & 150 [132-150] & 132 [115-146] & 120 [94-133] & 138 [119-150] & $p<0.001$ \\
\hline CK level (Ul/ml) & $\frac{150]}{112[60-460]}$ & $\begin{array}{l}550[123- \\
1500]\end{array}$ & $\begin{array}{l}780[249- \\
1332]\end{array}$ & $\begin{array}{l}586[296- \\
1123]\end{array}$ & 432 [109-974] & $P<0.001$ \\
\hline MDAAT (0-60) & $10[3.5-17]$ & $9[2-21.5]$ & na & na & $10[2.5-17]$ & 0.65 \\
\hline PGA (0-10) & $5[2-8]$ & $5[2-8]$ & $5[2-7]$ & na & $5[2-8]$ & 0.85 \\
\hline $\begin{array}{l}\text { Corticosteroids } \\
\text { n (\%) }\end{array}$ & $33(66)$ & $25(54)$ & $22(68)$ & 0 & $80(52)$ & 0.36 \\
\hline $\begin{array}{l}\text { Immunomodula } \\
\text { tor, } \mathrm{n}(\%)\end{array}$ & $23(46)$ & $26(57)$ & $17(53)$ & 0 & $66(43)$ & 0.58 \\
\hline
\end{tabular}

MMT8: manual muscle testing 8, CK: creatine phosphokinase, MDAAT: myositis disease activity assessment tool, na: non available, PGA: physician global assessment. Immunomodulator:

methotrexate, azathioprine, ciclosporine, rituximab, cyclophosphamide, hydroxychloroquine. MSA: myositis specific antibody (anti-Mi2, -Tif-1Y, -NXP2, -MDA5, -SAE1, -Jo1, -PL7, -PL12, -HMGCoa, -SRP, cn1a). MAA: myositis-associated antibody (anti-Ro52, -Ro60, RF, -CCP, -RNP, -DNA). DM:

Dermatomyositis, ASyS: Anti-synthetase syndrome, IMNM: Immune mediated necrotizing myopathie, IBM: Inclusion body myositis.

As expected, IBM patients were older and displayed a lower MMT8 score compared to DM and ASyS. MSA were detected in $70.6 \%$ of DM patients (anti-Mi2, $n=10 ;-$ TIF1 $\gamma, n=12 ;-N X P 2, n=7 ;-M D A 5, n=$ 5 and $-\mathrm{SAE}, \mathrm{n}=2$ ), by definition all ASyS were antibody positive (anti-Jo1, $\mathrm{n}=39 ;-\mathrm{PL7}, \mathrm{n}=4 ;-\mathrm{PL} 12, \mathrm{n}$ $=2$ and - EJ, $n=1$ ), and all IMNM patients were seropositive (anti-SRP, $n=13,-H M G C R, n=19$ ).

No difference was observed in the therapeutic profile, including the use of corticosteroids and immunosuppressors, between IMNM, DM, and ASyS while IBM patients did not receive any treatment. Increased levels of type I and II IFNs depend on the myositis subgroups Serum IFN- $\alpha$ level was significantly higher in DM $(0.07[0.03-0.23] \mathrm{pg} / \mathrm{ml})$ and ASyS $(0.07[0.02-0.16]$ $\mathrm{pg} / \mathrm{ml})$ compared to HD $(0.02[0.01-0.05] \mathrm{pg} / \mathrm{ml} ; \mathrm{p}<0.005$ and $\mathrm{p}<0.05$ respectively) whereas it was not significantly different in IMNM $(0.03[0.01-0.09] \mathrm{pg} / \mathrm{ml})$ or IBM $(0.02[0.02-0.03] \mathrm{pg} / \mathrm{ml})$ compared to HD (Fig. 1a). One quarter ( $26 \% ; n=13 / 50)$ of DM and $20 \%$ ASyS patients $(n=9 / 46)$ had increased IFN- $\alpha$ level above the positivity threshold while only $3 \%$ of IMNM $(n=1 / 32)$ and $4 \%$ of IBM patients ( $n$ $=1 / 24$ ) had increased levels.

Only DM patients had significantly higher IFN- $\beta$ level (1.24 [1.24-6.31] pg/ml) compared to HD (1.24 
[1.24-1.24] pg/ml, $p<0.005)$ (Fig. 1b). IFN- $\beta$ was increased in 34\% ( $n=17 / 50)$ of DM patients, and $12 \%$ of IBM patients $(n=3 / 24)$ while no ASyS or IMNM patients presented an increased level.

IFN-ץ level was significantly increased in all IIM subgroups (ASyS (1.05 [0.47-2.46] pg/ml), DM (0.90 [0.55-2.09] pg/ml), IMNM (0.96 [0.42-1.29] pg/ml) and IBM (0.93 [0.42-2.09] pg/ml)) compared with HD (0.46 [0.29-0.59] pg/ml), $p<0.05)$ (Fig. 1c). One third of ASyS patients (37\%; $n=17 / 46)$, one quarter of DM patients $(26 \% ; n=13 / 50)$ and IBM patients $(25 \%, n=6 / 24)$ and $16 \%$ of IMNM patients ( $n=5 / 32)$ had an increased level of IFN-Y.

\section{IFN levels and disease activity}

Type I and II IFN levels are correlated with disease activity of DM and ASyS

Correlation between IFN level and disease activity showed that disease activity was strongly correlated with type-I IFN, IFN- $\alpha(r=0.76[0.60-0.86], p<0.001)$ (Fig. $2 a)$ and IFN- $\beta(r=0.58[0.35-$ 0.74], $p<0.01$ ) (Fig. 2c) in DM. A mild correlation with IFN- $\gamma(r=0.36$ [0.05-0.56], $p=0.02)$ was observed.

ASyS also demonstrated that disease activity correlated with IFN- $\alpha(r=0.55[0.34-0.76], p<0.001)$ (Fig. 2e) and IFN- $\gamma$ levels ( $r=0.46$ [0.15-0.66], $p=0.003$ ) (Fig. $2 g$ ). Of note, no ASyS patient presenting an active disease had increased IFN- $\beta$ level. In IMNM, only IFN- $\gamma$ level was significantly correlated with disease activity $(r=0.48$ [0.14-0.71], $p=$ $0.006)$ whereas IFN- $\alpha(r=0.23[-0.14-0.55], p=0.2)$ and IFN- $\beta(r=-0.07[-0.43-0.31], p=0.7)$ were not. Of note, correlation between CK levels and disease activity was very high $(r=0.87$ [0.73-0.94], $p$ $<0.001$ ) for IMNM patients.

Multivariate analysis including IFN- $\alpha$, and IFN- $\gamma$ showed that only IFN- $\alpha$ was associated with active disease in DM patients (OR $=9.5$ [3.1-45.9], $\mathrm{p}<0.001)$. Concerning ASyS patients, only IFN- $\alpha$ was statistically associated with disease activity $(O R=5[1.9-17.9], p=0.004)$, and there was a trend for IFN- $Y(p=0.08)$. No IFN subtype was associated with disease activity in the IMNM subgroup. Longitudinal analysis

Focusing on DM and ASyS treatment-naive patients at diagnosis, we performed a serial longitudinal analysis (DM, $n=6 / 11$ and ASyS, $n=4 / 11$ ) (supplementary Fig. 1 and Fig. 3). The majority of DM 
patients had a high type I IFN level at diagnosis and a decrease parallel to the clinical improvement (Fig. 3a, Fig. 3b and supplementary Fig. 1). IFN-y level wasn't associated with the disease activity in follow-up of DM (Fig. 3c). In most ASyS patients increased levels of IFN- $\alpha$ and IFN- $\gamma$ but not IFN- $\beta$ were observed at the diagnosis (Supplementary Fig. 2). Similarly, these levels decreased following the clinical improvement.

Sensitivity and specificity of IFNs to discriminate active and inactive DM and ASyS patients Next, we aimed to define the threshold level of IFN corresponding to active disease. Active DM patients had higher level of IFN- $\alpha(0.26[0.09-0.53] \mathrm{pg} / \mathrm{ml})$ compared to inactive DM patients $(0.03$ [0.01-0.07] pg/ml, p < 0.001) (Supplementary Fig. 2a). ROC analysis showed that the area under the curve (AUC) was 0.88 (IC95 0.79-0.98; p < 0.001) (Fig. 2b). An IFN- $\alpha$ threshold above $0.11 \mathrm{pg} / \mathrm{ml}$ had a $75 \%$ sensitivity and $96.2 \%$ specificity, and 19.5 positive likelihood ratio to discriminate active from inactive DM patients (Table 2).

Table 2

Sensitivity and specificity of different IFNs as activity biomarkers

\begin{tabular}{|c|c|c|c|c|}
\hline & IFN level (pg/ml) & Se (\%) & Sp (\%) & Likelihood ratio + \\
\hline DM IFN- $\alpha$ & 0.11 & 75 & 96 & 19.50 \\
\hline IFN- $\beta$ & 1.6 & 57 & 88 & 4.90 \\
\hline IFN- $\gamma$ & 1.06 & 71 & 77 & 3.07 \\
\hline ASyS IFN- $\alpha$ & 0.06 & 82 & 81 & 4.42 \\
\hline IFN- & 1.05 & 80 & 73 & 2.97 \\
\hline
\end{tabular}

DM: Dermatomyositis, ASyS: Anti-synthetase syndrome, IFN: Interferon, Se : sensitivity, Sp : Specificity.

Active DM patients had higher level of IFN- $\beta$ (4.62 [1.24.-27.96]) compared to inactive DM patients (1.24 [1.24-1.24] pg/ml, $p=0.0001$ ) (Supplementary Fig. 2b) with a 0.75 AUC (IC95 0.61-0.89; $p=$ 0.0027) (Fig. 2d). Of note, IFN-y levels were higher in active DM patients (1.417 [0.81-2.74] pg/ml) compared to inactive ones $(0.64[0.38-1.20] \mathrm{pg} / \mathrm{ml}, \mathrm{p}=0.007)$.

Active ASyS patients had higher IFN- $\alpha$ level $(0.16[0.08-0.36] \mathrm{pg} / \mathrm{ml})$ compared to inactive ASyS patients $(0.03[0.01-0.06] \mathrm{pg} / \mathrm{ml}, \mathrm{p}<0.001)$ (Supplementary Fig. $2 \mathrm{c}$ ). The sensitivity was $82.3 \%$ and the specificity $80.8 \%$ for an IFN- $\alpha$ threshold above $0.06 \mathrm{pg} / \mathrm{ml}(\mathrm{AUC}=0.86, \mathrm{IC} 95(0.74-0.97) ; \mathrm{p}<$ 0.001) (Fig. 2f). Active ASyS patients had higher level of IFN-Y (2.28 [1.18-3.26] pg/ml) compared to inactive ASyS patients (0.82 [0.34-1.33] pg/ml, $\mathrm{p}=0.004)$ (Supplementary Fig. $2 \mathrm{~d}$ ) and sensitivity and 
specificity were $80 \%$ and $73.1 \%$ respectively at a threshold above $1.05 \mathrm{pg} / \mathrm{ml}$ (Table 2 ) (AUC $=0.75$, IC95(0.60-0.90); $p=0.003$ (Fig. 2 h)).

\section{Discussion}

In this study, we showed that type I IFN is a reliable biomarker of disease activity in DM and ASyS patients. While IFN- $\alpha$ is increased in both conditions, IFN- $\beta$ is only increased in DM patients. Type II IFN (i.e IFN-Y) is increased in all myositis subgroups but ASyS patients showed the best correlation with the disease activity.

Previous studies have described an IFN signature in muscle, skin and blood samples of DM patients (20-22). The IFN-stimulated genes levels can be assessed by an IFN score (6) combining a set of at least five genes, but it is not standardized and performed routinely in clinical practice, and requires RNA extraction. The IFN score in DM was only correlated with the cutaneous disease activity (9). Digital ELISA for type I IFN is more sensitive than ELISA and was very well correlated with IFN-gene signature $(9,17)$. For the first time, we were able to show a very good correlation with DM disease activity parameters and IFN-I levels. This technology was recently used in lupus with interesting data (19). Only one previous study used Digital ELISA to measure IFN- $\alpha$ blood level in adult DM (9). It did not show a significant correlation with disease activity but in this study, only the skin disease activity was assessed whereas we included all domains of disease activity (e.g skin, muscle and joints). In this study, IFN- $\beta$ only was correlated with the skin disease activity (9) in line with a previous study showing that IFN- $\beta$ was increased in DM (8). In our study, we included a large cohort of myositis patients and we confirmed that IFN- $\beta$ is a DM specific IFN cytokine and showed for the first time that both IFN- $\alpha$ and $\beta$ are reliable biomarkers. Nevertheless, we have to underline that the limit of detection of IFN- $\alpha$ was 300 times lower than for IFN- $\beta$ it might be possible that interest of IFN- $\beta$ level monitoring will increase when the limit of detection will be improved.

In addition, for the first time, we demonstrated that IFN- $\alpha$ is also associated with disease activity in ASyS. Along that line, it was previously shown that anti-Jol positive patients harbored an IFN signature (23). If both DM and ASyS have an IFN signature, we observed that only DM have increased level of IFN- $\beta$ and that IFN- $\gamma$ was a good biomarker in ASyS but not in DM. It was shown that only DM 
patients expressed in the muscles IFN-related proteins $(24,25)$ while muscle fibers of ASyS overexpressed MHC-II (26), a type II IFN inducible protein (27). Along that line, a recent study showed that different IFN signature is found in muscle biopsy of IIM (28). Altogether these results highlight that pathways in both conditions are different: namely IFN- $\alpha$ and $-\beta$ in DM and IFN- $\alpha$ and $-\gamma$ in ASyS. In IMNM, only IFN-Y was associated with disease activity but CK level was a better biomarker according to a previous study showing a strong correlation between the percentage of necrotic muscle fibers and CK level (4). Increased type II IFN levels may be due to Th-1 immune responses and $\mathrm{CD}^{+} \mathrm{T}$ cells or NK cells, two types of immune cells involved in pathophysiology of IBM and ASyS respectively $(10,29)$, but also by macrophages $(17)$, the most abundant cells infiltrating IMNM muscle (4).

DM and ASyS are two multisystemic conditions making disease activity assessment very complex. In this study, we demonstrated that IFNs are reliable biomarkers of disease activity. There is no gold standard to measure myositis disease activity and we cannot exclude that monitoring IFN levels is more sensitive to assess disease activity than current clinical tools (3). We acknowledge that we were unable to calculate the improvement score as recommended by the ACR/EULAR to assess patients' disease activity at two timepoints. Similarly, patients' reported outcomes such as health assessment questionnaire or patient visual analog scale were unavailable.

\section{Conclusion}

To conclude, this study showed that type I and II IFNs are reliable biomarkers for DM and ASyS disease activity and further independent prospective studies are required to confirm these results.

\section{Abbreviations}

Anti-synthetase syndrome: AsyS

Creatine kinase: CK

Dermatomyositis: DM

Immune-mediated necrotizing myopathy: IMNM

Inclusion body myositis: IBM

Inflammatory idiopathic myopathies: IIM 
Interferon: IFN

Limit of detection: LOD

Manual Muscle Testing 8: MMT8

Myositis-Specific Antibody: MSA

Myositis Disease Activity Assessment Tool: MDAAT

Physician Global Activity: PGA

Declarations

Ethics approval and consent to participate

Written informed consent from each study patient and approval by local Ethics Committee (CPP Ile De France VI (2013-12-19), CCTIRS (N¹4.323) and CNIL (AR158656)) were obtained. Patient were not involved in the design and conduct of our research

Consent for publication

Patient consent for publication were not required.

Availability of data and materials

All data relevant to the study are included in the article or uploaded as supplementary information.

Data are available upon reasonable request.

Competing interests

All the authors declare no disclosure.

Funding

Authors declare no funding.

Authors' contributions

LB, YA, OB and CA were involved in the study design. LB, DA, KD and GG realized IFN alpha and gamma measurement. LB, AL, VB and DD were involved in IFN beta measurement and validation of the test. LB, CA and KM realized statistical analysis. LB, ST, OLC, AM and BH realized clinical evaluation.

Acknowledgements

We Acknowledge all the doctors for the clinical information: A. Rigolet, P. Guillaume-Jugnot, M. 
Vautier, N. Champtiaux, G. Leroux, P. Cacoub, F. Domont, D. SAADOUN, N. Sbeih.

\section{References}

1. Mariampillai K, Granger B, Amelin D et al. Development of a New Classification System for Idiopathic Inflammatory Myopathies Based on Clinical Manifestations and Myositis-Specific Autoantibodies. JAMA Neurol. 10 sept 2018;

2. Selva-O'Callaghan A, Pinal-Fernandez I, Trallero-Araguás E et al. Classification and management of adult inflammatory myopathies. Lancet Neurol. sept $2018 ; 17(9): 816-28$.

3. Aggarwal R, Rider LG, Ruperto $\mathrm{N}$ et al. 2016 American College of Rheumatology/European League Against Rheumatism criteria for minimal, moderate, and major clinical response in adult dermatomyositis and polymyositis: An International Myositis Assessment and Clinical Studies Group/Paediatric Rheumatology International Trials Organisation Collaborative Initiative. Ann Rheum Dis. mai 2017;76(5):792-801.

4. Allenbach $\mathrm{Y}$, Arouche-Delaperche L, Preusse $\mathrm{C}$ et al. Necrosis in anti-SRP+ and antiHMGCR+myopathies: Role of autoantibodies and complement. Neurology. 6 févr 2018;90(6):e507-17.

5. Mathur T, Manadan AM, Thiagarajan S et al. The utility of serum aldolase in normal creatine kinase dermatomyositis. J Clin Rheumatol Pract Rep Rheum Musculoskelet Dis. janv 2014;20(1):47-8.

6. Greenberg SA, Pinkus JL, Pinkus GS et al. Interferon-alpha/beta-mediated innate immune mechanisms in dermatomyositis. Ann Neurol. mai 2005;57(5):664-78.

7. Greenberg SA, Higgs BW, Morehouse C et al. Relationship between disease activity and type 1 interferon- and other cytokine-inducible gene expression in blood in dermatomyositis and polymyositis. Genes Immun. avr 2012;13(3):207-13. 
8. Liao AP, Salajegheh $M$, Nazareno $R$ et al. Interferon $\beta$ is associated with type 1 interferon-inducible gene expression in dermatomyositis. Ann Rheum Dis. mai $2011 ; 70(5): 831-6$.

9. Huard C, Gullà SV, Bennett DV et al. Correlation of cutaneous disease activity with type 1 interferon gene signature and interferon $\beta$ in dermatomyositis. Br J Dermatol. mai $2017 ; 176(5): 1224-30$.

10. Allenbach Y, Chaara W, Rosenzwajg M et al. Th1 response and systemic treg deficiency in inclusion body myositis. PloS One. 2014;9(3):e88788.

11. Greenberg SA, Pinkus JL, Amato AA et al. Association of inclusion body myositis with T cell large granular lymphocytic leukaemia. Brain J Neurol. 2016;139(Pt 5):1348-60.

12. Wilson DH, Rissin DM, Kan CW et al. The Simoa HD-1 Analyzer: A Novel Fully Automated Digital Immunoassay Analyzer with Single-Molecule Sensitivity and Multiplexing. J Lab Autom. août 2016;21(4):533-47.

13. Lundberg IE, Tjärnlund A, Bottai M et al. 2017 European League Against Rheumatism/American College of Rheumatology Classification Criteria for Adult and Juvenile Idiopathic Inflammatory Myopathies and Their Major Subgroups. Arthritis Rheumatol Hoboken NJ. 2017;69(12):2271-82.

14. Lloyd TE, Mammen AL, Amato AA, et al. Evaluation and construction of diagnostic criteria for inclusion body myositis. Neurology. 29 juill 2014;83(5):426-33.

15. Allenbach Y, Mammen AL, Benveniste O et al. Immune-Mediated Necrotizing Myopathies Working Group. 224th ENMC International Workshop:: Clinico-seropathological classification of immune-mediated necrotizing myopathies Zandvoort, The Netherlands, 14-16 October 2016. Neuromuscul Disord NMD. janv $2018 ; 28(1): 87-99$.

16. Flammer JR, Dobrovolna J, Kennedy MA et al. The type I interferon signaling pathway 
is a target for glucocorticoid inhibition. Mol Cell Biol. oct 2010;30(19):4564-74.

17. Rodero MP, Decalf J, Bondet $V$ et al. Detection of interferon alpha protein reveals differential levels and cellular sources in disease. J Exp Med. 1 mai $2017 ; 214(5): 1547-55$.

18. Cohen L, Xie L, Xylas ME et al. Single Molecule Arrays for ultra-sensitive detection of rat cytokines in serum. J Immunol Methods. 2018;452:20-5.

19. Mathian A, Mouries-Martin S, Dorgham K et al. Monitoring disease activity in systemic Iupus erythematosus with single-molecule array digital ELISA quantification of serum interferon- $\alpha$. Arthritis Rheumatol. 3 déc 2018;

20. Allenbach Y, Leroux G, Suárez-Calvet X et al. Dermatomyositis With or Without AntiMelanoma Differentiation-Associated Gene 5 Antibodies: Common Interferon Signature but Distinct NOS2 Expression. Am J Pathol. mars 2016;186(3):691-700.

21. Salajegheh M, Kong SW, Pinkus JL et al. Interferon-stimulated gene 15 (ISG15) conjugates proteins in dermatomyositis muscle with perifascicular atrophy. Ann Neurol. janv 2010;67(1):53-63.

22. Rice GI, Forte GMA, Szynkiewicz M et al. Assessment of interferon-related biomarkers in Aicardi-Goutières syndrome associated with mutations in TREX1, RNASEH2A, RNASEH2B, RNASEH2C, SAMHD1, and ADAR: a case-control study. Lancet Neurol. déc 2013;12(12):1159-69.

23. Ekholm L, Vosslamber S, Tjärnlund A et al. Autoantibody Specificities and Type I Interferon Pathway Activation in Idiopathic Inflammatory Myopathies. Scand J Immunol. août 2016;84(2):100-9.

24. Uruha A, Nishikawa A, Tsuburaya RS et al. Sarcoplasmic MxA expression: A valuable marker of dermatomyositis. Neurology. 31 2017;88(5):493-500.

25. Uruha A, Allenbach Y, Charuel J-L et al. Diagnostic potential of sarcoplasmic MxA 
expression in subsets of dermatomyositis. Neuropathol Appl Neurobiol. 28 sept 2018;

26. Aouizerate J, De Antonio M, Bassez G et al. Myofiber HLA-DR expression is a distinctive biomarker for antisynthetase-associated myopathy. Acta Neuropathol Commun. 23 oct $2014 ; 2: 154$.

27. Rigolet M, Hou C, Baba Amer Yet al. Distinct interferon signatures stratify inflammatory and dysimmune myopathies. RMD Open. 2019;5(1):e000811.

28. Pinal-Fernandez I, Casal-Dominguez M, Derfoul A et al. Identification of distinctive interferon gene signatures in different types of myositis. Neurology. 17 sept 2019;93(12):e1193-204.

29. Hervier B, Perez M, Allenbach Y et al. Involvement of NK Cells and NKp30 Pathway in Antisynthetase Syndrome. J Immunol Baltim Md 1950. 01 2016;197(5):1621-30.

\section{Supplementary Figures}

Supplementary figure 1: IFN level in naive patients at diagnosis. a IFN- $\alpha$ at diagnosis in IIM patients b IFN- $\beta$ at diagnosis in IIM patients c IFN-Y at diagnosis in IIM patients. The dot line represents the mean +3 standard deviations (positivity threshold).

\section{Supplementary figure 2: Comparison between active (PGA $>5$ ) and inactive patients}

(PGA 5). a IFN- $\alpha$ in active and inactive DM patients $\mathbf{b}$ IFN- $\beta$ in active and inactive DM patients $\mathbf{c}$ IFN$\alpha$ in active and inactive ASyS patients d IFN- $\gamma$ in active and inactive ASyS patients. $* * p<0.005$ DM: Dermatomyositis, ASyS: Anti-synthetase syndrome,

Figures 

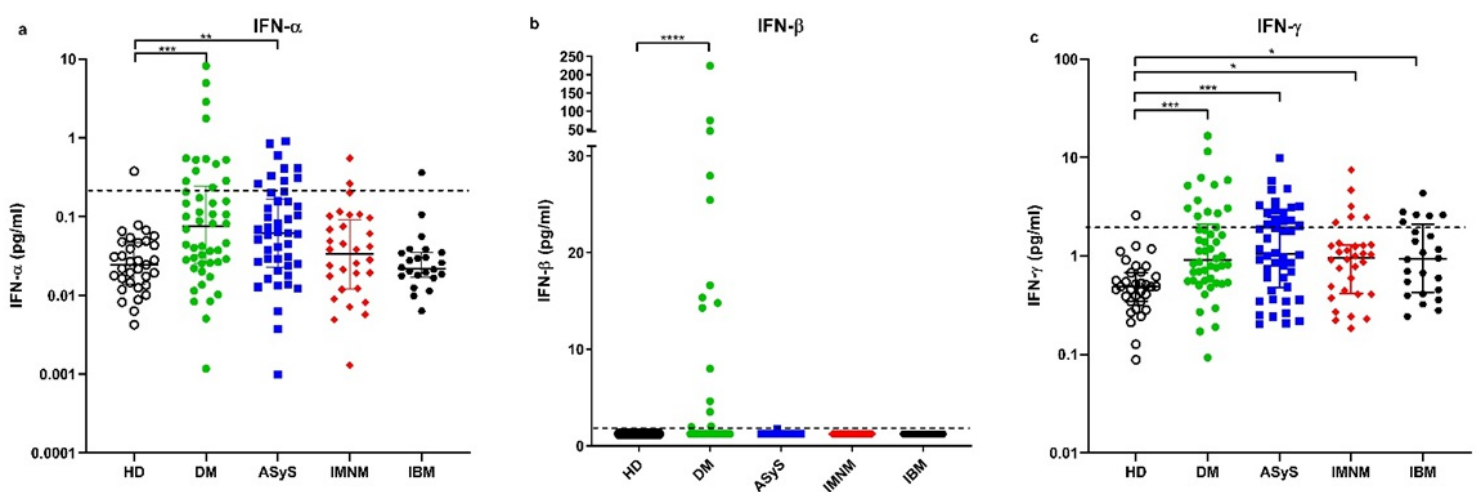

Figure 1

IFN levels in IIM. DM: Dermatomyositis, ASyS: Anti-synthetase syndrome, IMNM: Immunemediated necrotizing myopathies, IBM: Inclusion body myositis, HD: healthy donors - - mean \pm 3 standard deviation or positivity threshold, $*: p<0.05, * * p<0.005, * * *: p<0.0005, * * * *$

$$
p<0.00005
$$



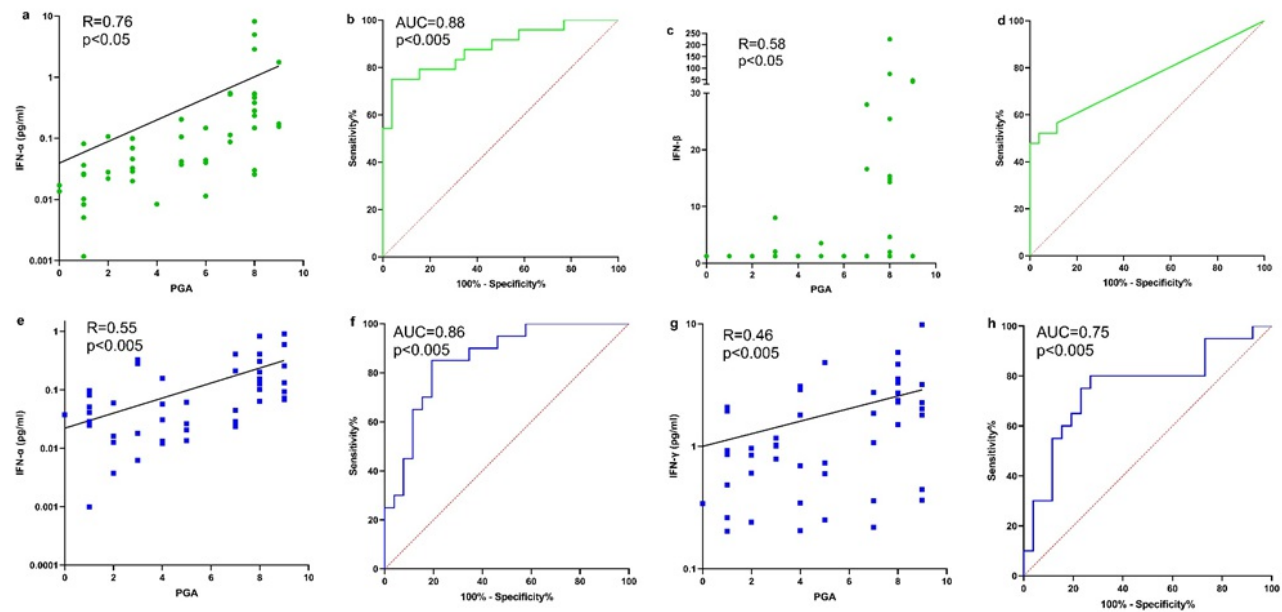

Figure 2

Correlation between IFNs and disease activity. Correlation between IFN- $\alpha$ and DM disease activity. b ROC curve concerning IFN- $\alpha$ level between active DM patients (PGA>5) and nonactive DM patients (PGA $\leq 5$ ). c Correlation between IFN- $\beta$ and DM disease activity. $d$ ROC curve concerning IFN- $\beta$ level between active DM patients (PGA>5) and non-active DM patients e Correlation between IFN- $\alpha$ and ASyS disease activity. $f$ ROC curve concerning IFN$\alpha$ level between active ASyS patients (PGA $>5$ ) and non-active ASyS patients (PGA $\leq 5) . g$ Correlation between IFN- $\gamma$ and ASyS disease activity. h ROC curve concerning IFN- $\gamma$ level between active ASyS patients (PGA $>5$ ) and non-active ASyS patients (PGA $\leq 5)$ DM:

Dermatomyositis, ASyS: Anti-synthetase syndrome, AUC: area under the curve, PGA: physician global activity. 

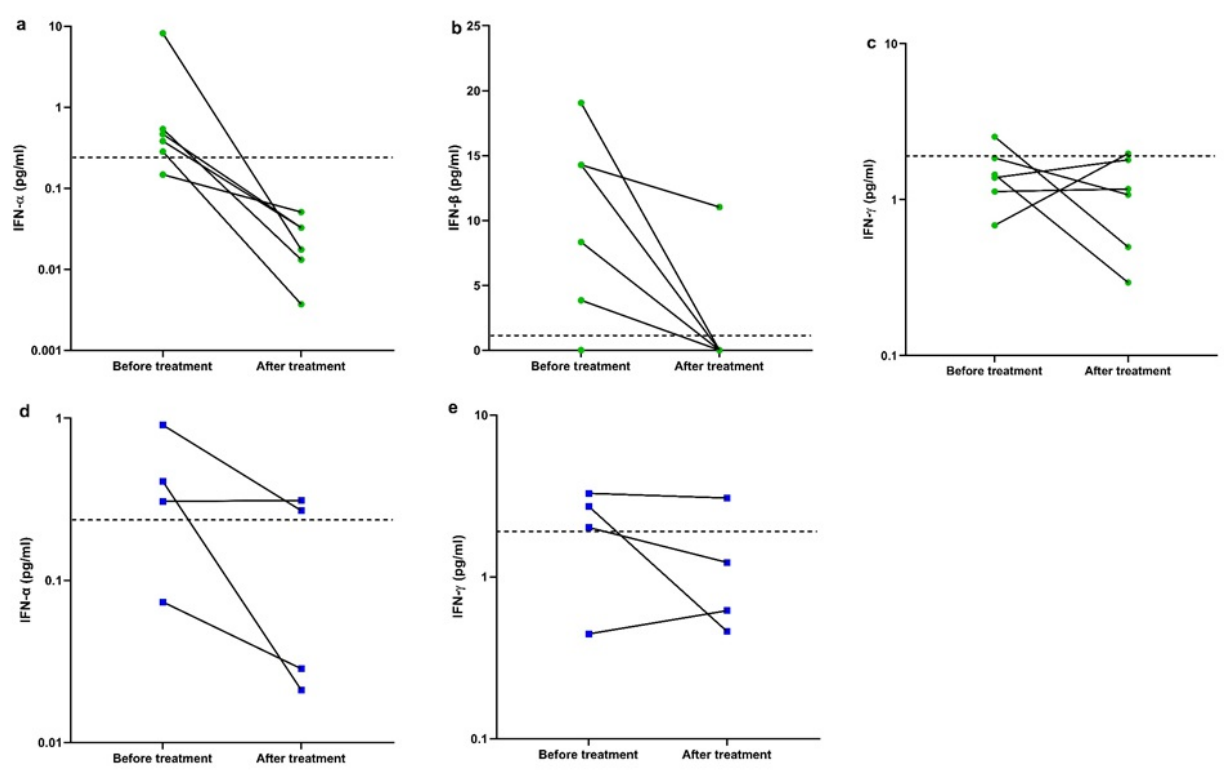

Figure 3

Evolution of IFNs levels following treatment. a Evolution of IFN- $\alpha$ in DM patients. b Evolution of IFN- $\beta$ in DM patients. $c$ Evolution of IFN- $\gamma$ in DM patients. $d$ Evolution of IFN- $\alpha$ in ASyS patients. e Evolution of IFN- $\gamma$ in ASyS patients. The dot line represents the mean +3 standard deviations (positivity threshold). DM: Dermatomyositis, ASyS: Anti-synthetase syndrome,

\section{Supplementary Files}

This is a list of supplementary files associated with this preprint. Click to download.

Supplementary figures.pptx 